\title{
SEMICONTINUOUS PLANAR TOTAL PREORDERS ON NON-SEPARABLE METRIC SPACES
}

\author{
María Jesús Campión, Juan Carlos Candeal, and Esteban Induráin
}

\begin{abstract}
We prove that every non-separable connected metric space can be endowed with a total preorder that is order-isomorphic to a nonrepresentable subset of the lexicographic plane and semicontinuous with respect to the metric topology.
\end{abstract}

\section{Introduction}

The main purpose of this paper is to prove that in a wide class of nonseparable metric spaces, that includes the connected ones, it is possible to construct total preorders that are order-isomorphic to a non-representable subset of the lexicographic plane and semicontinuous with respect to the metric topology.

As analyzed in Beardon et al. [5] (see also Giarlotta [23, 24]), there are three different classes of totally ordered structures that cannot be represented in the real line through an order-isomorphism.

These classes are: (i) long chains, i.e., chains containing a subchain orderisomorphic to the first uncountable ordinal, (ii) Aronszajn chains, i.e., uncountable chains that neither are long, nor contain any uncountable representable subchain, (iii) planar chains, i.e., chains containing a subchain order-isomorphic to a non-representable subset of the lexicographic plane.

Although these three classes appeared formerly for total orders, this classification can be extended to total preorders because, through the indifference relation, we obtain a totally ordered structure in the quotient. And this totally ordered quotient is representable if and only if the given total preorder is.

Given a topological space $(X, \tau)$, a topological question related with ordered structures consists in deciding if $X$ can be given a total preorder that is nonrepresentable and continuous (or semicontinuous) with respect to the given

Received August 20, 2007.

2000 Mathematics Subject Classification. Primary 54F05, Secondary 06A06, 54C08, $54 \mathrm{C} 30$.

Key words and phrases. real-valued functions, metric spaces, semicontinuous total preorders, representability. 
topology $\tau$ on $X$. This is the continuous representability problem (or semicontinuous representability problem), see Herden and Pallack [25] and Bosi and Herden [9]. Thus, a topological space $(X, \tau)$ is said to satisfy the continuous representability property $\mathrm{CRP}$, (respectively, the semicontinuous representability property SRP) if every $\tau$-continuous (respectively $\tau$-semicontinuous) total preorder $\precsim$ defined on $X$ admits a representation by means of a real-valued order-preserving map $U:(X, \tau) \rightarrow(\mathbb{R}$, Euclidean topology) (i.e.,: $x \precsim y \Longleftrightarrow$ $U(x) \leq U(y)(x, y \in X))$, that is continuous (respectively, semicontinuous). In other words, the continuous representability problem (respectively, the semicontinuous representability problem) consists in deciding if $(X, \tau)$ satisfies CRP or not (respectively, satisfies SRP or not). It can be proved that SRP always implies CRP, but the converse is not true in general (see e.g. Bosi and Herden $[9])$.

These problems are related to other important topological questions, namely the extension of continuous orderings from a subset to the whole set (see e.g. Yi [34], Bosi-Herden [10, 11], and Campión et al. [14]). It has also connections with structural properties of classical topological spaces arising in functional analysis (e.g. Topological vector spaces, and, in particular, Banach spaces), see Candeal et al. [20], Campión et al. [13, 15].

In this direction there is a nice result, due to Estévez and Hervés [22], for the particular case of metric spaces:

In any non-separable metric space it is possible to construct a continuous total preorder that does not admit a representation by means of a real-valued order-preserving function.

(Observe that this result says that non-separable metric spaces never satisfy CRP. As a matter of fact, it is not difficult to prove that separable metric spaces do satisfy CRP, see e.g., Candeal et al. [16]).

The key to prove this important result is based on the existence of a continuous function from the metric space onto the first uncountable ordinal number, and as a matter of fact such total preorders defined in Estévez-Hervés [22] are long. Other examples of continuous and non-representable total preorders, defined on particular subsets of infinite-dimensional topological vector spaces, had already been considered in the literature (see, e.g., Candeal et al. [20], Monteiro [29] or Bosi et al. [8]). However, those examples make use of the first uncountable ordinal number, and belong to the class of long total preorders.

Up to date, on metric spaces no general examples of either continuous or semicontinuous non-representable total preorders of the aforementioned other classes (planar or Aronszajn) are known.

In fact, a new topological classification for general metric spaces (even for general topological spaces, metric or not!) can be introduced now, in the spirit of the example in Estévez-Hervés [22]. Thus, a topological space $(X, \tau)$ is said to be singular of the first (respectively second, third) type with respect to continuous ordinal representability if it does not satisfy CRP and can be endowed with a non-representable continuous total preorder that is long (respectively 
Aronszajn, planar). Thus, the result in Estévez-Hervés can be rephrased by saying that every nonseparable metric space is singular of the first type (long) with respect to continuous ordinal representability.

On the other hand, it is also well-known ${ }^{1}$ (see e.g. Bridges and Mehta [12], Theorem 3.2.7 on p. 47, and Isler [26]) that:

Any semicontinuous total preorder defined on a second countable topological space can be represented by a semicontinuous real-valued order-preserving function.

In other words, this result states that every second countable topological space satisfies SRP. The converse is not true, in general: An example is the separable Banach space $\ell_{2}(\mathbb{N})$ endowed with the weak topology. It satisfies SRP as a consequence of Theorem 3.1 in Campión et al [14]. On the other hand, the weak topology on this space does not satisfy the second axiom of countability (not even the first axiom of countability), because this Banach space has infinite dimension (see Theorem 6.30 on pp. 254-255 of Aliprantis and Border [1] for details).

As a direct consequence, neither continuous nor even semicontinuous nonrepresentable total preorders can be defined on second countable topological spaces.

Moreover, since in metric spaces second countability and separability are equivalent concepts, we see that the condition of non-separability cannot be dropped from the statement of the result in Estévez-Hervés on the possibility of constructing continuous non-representable total preorders on metric spaces. Indeed, the equivalence between second countability and separability on metric spaces has an important consequence: A metric space satisfies CRP if and only if it satisfies SRP. (See e.g. Campión et al. [14] for further details.)

As defined before, but now involving semicontinuity, we say that a topological space $(X, \tau)$ is singular of the first (respectively second, third) type with respect to semicontinuous ordinal representability if it does not satisfy SRP and can be endowed with a non-representable semicontinuous total preorder that is long (respectively Aronszajn, planar). Observe, in addition that if a topological space is singular of the first (respectively second, third) type with respect to continuous ordinal representability, then it is also singular of the same type with respect to semicontinuous ordinal representability. The converse is not true, not even for metric spaces, as we prove later.

In the present paper we will prove that semicontinuous non-representable total preorders of the planar class can be constructed on any non-separable connected metric space. In other words, any non-separable connected metric space is singular of the third type (planar) with respect to semicontinuous ordinal representability. (For an account of the lexicographic behaviour of general

\footnotetext{
${ }^{1}$ This classical result is known in the literature as Rader's theorem, see Rader [31]. However, the original proof of this result had a flaw that was corrected in later works, e.g. in Isler [26].
} 
totally ordered sets, see Candeal and Induráin [18]. For further information on particular features of the structure of planar chains, consult Beardon et al. [6]. Some particular planar chains have also been studied in Ostaszewski [30], Steen and Seebach [33], p. 73, or Giarlotta [23].)

Moreover, we shall also prove that on path connected metric spaces semicontinuity cannot be replaced by continuity if we are looking for non-representable total preorders belonging to the planar class. In other words, no continuous non-representable total preorders of the planar class can be defined on a pathconnected metric space. Therefore, path-connected metric spaces cannot be singular of the third type (planar) with respect to continuous ordinal representability, despite they always are singular of the planar type with respect to semicontinuous ordinal representability as we prove below.

To conclude this introduction, we would like to point out some possible applications of the concepts that we shall study in the present paper:

Thus, in some contexts arising in Economic Theory, the consumption sets to deal with are typically non-separable metric spaces (see Aliprantis et al. [2] and Mas-Colell and Zame [28]). This happens if the commodity space is $L^{\infty}$, the space of all essentially bounded measurable functions defined on a $\sigma$-finite measure space, which arises in studies concerning allocation of resources over time or states of nature (see e.g. Bewley [7]), or ca(K), the space of countably additive signed measures on a compact metric space which is crucial for the study of commodity differentiation (see e.g. Mas-Colell [27]). Some appealing recent approaches to general equilibrium theory in infinite-dimensional commodity spaces are based on the extension of the arguments of the second welfare theorem (that holds for the finite-dimensional case) to the infinite-dimensional setting. These, in particular, postulate the existence of the utility possibility set, which obviously depends on the existence of utility functions (that are real-valued order-preserving representations of a total preorder) for the preference relations (that are total preorders defined on the consumption set) of each individual. From this point of view, it should be desirable to have utility representation results (that is, results characterizing the representability of total preorders through real-valued order-preserving functions) in metric or quasimetric spaces (see Romaguera and Sanchis [32], where some other applications to Theoretical Computer Science are also commented), and consequently, to know which particular cases of non-representable preference relations could appear in such approach. The results that will be obtained in the present paper apply to those particular cases of non-separable metric spaces.

\section{Preliminaries}

By $(X, d)$, or simply by $X$, we will denote a topological metric space, i.e., a set endowed with a topology that is given by a metric $d$. Given $x \in X$ and $\epsilon>0$ we will denote by $B(x, \epsilon)$ the closed ball centered at $x$ with radius $\epsilon$, i.e., $B(x, \epsilon)=\{y \in X ; d(x, y) \leq \epsilon\}$. 
A metric space $(X, d)$ is called full if for each $x \in X$ the range of the continuous map $d(x,):. X \longrightarrow \mathbb{R}$ is a connected subset of the real line. (In other words: if for every $x, y \in X$ and $\delta \in \mathbb{R}$ with $0<\delta<d(x, y)$, there is $z \in X$ such that $d(x, z)=\delta)$.

Let $\preceq$ be a total preorder defined on $X$; that is, a reflexive, transitive and total binary relation defined on $X$. If, in addition, $\preceq$ is antisymmetric, then $\preceq$ is said to be a total order. For any total preorder $\preceq$ defined on $X$ we may associate both an asymmetric and negatively transitive binary relation $\prec$ defined by $x \prec y \Longleftrightarrow(x \preceq y)$ and not $(y \preceq x)$, and an equivalence relation $\sim$, called the indifference relation, defined as $x \sim y \Longleftrightarrow(x \preceq y)$ and $(y \preceq x)$. Note that $\preceq$ induces on the quotient space $X / \sim$ a total order, defined in the obvious way.

In the particular case in which $\preceq$ is a total order on $X$, the pair $(X, \prec)$ is said to be a chain.

Given $x, y \in X, x \prec y$, we denote by $(x, y)$ the order interval $(x, y)=\{z \in$ $X ; x \prec z \prec y\}$.

Let $\left(S_{1}, \preceq_{1}\right), \quad\left(S_{2}, \preceq_{2}\right)$ be two totally preordered spaces. A function $u$ : $S_{1} \longrightarrow S_{2}$ is said to be order-preserving if $s_{1} \preceq_{1} s_{1}^{\prime}$ if and only if $u\left(s_{1}\right) \preceq_{2} u\left(s_{1}^{\prime}\right)$ for every $s_{1}, s_{1}^{\prime} \in S_{1}$. If $u$ is order-preserving and onto, then $\left(S_{1}, \preceq_{1}\right),\left(S_{2}, \preceq_{2}\right)$ are said to be order-isomorphic.

If $\left(S_{2}, \preceq_{2}\right)$ is a subset of the reals, endowed with the usual order, an orderisomorphism $u: S_{1} \longrightarrow S_{2} \subseteq \mathbb{R}$ is also called a utility function.

The lexicographic plane, denoted by $\left(\mathbb{R}^{2}, \leq_{l}\right)$, is meant to be the plane $\mathbb{R}^{2}$ equipped with the lexicographic total order defined as: $(x, y) \leq_{l}(u, v) \Leftrightarrow x<u$ or $x=u, y \leq v$.

A total preorder $\preceq$ defined on $X$ is said to be:

- representable (it is also said that the structure $(X, \preceq)$ is representable) if there exists a real-valued order-isomorphism $U: X \longrightarrow U(X) \subseteq \mathbb{R}$;

- order separable if there is a countable subset $D \subseteq X$ such that for every $x, y \in X, x \prec y$, there is $d \in D$ such that $x \preceq d \preceq y$;

- countably bounded if there is a countable subset $D \subseteq X$ that bounds $\preceq$, i.e., for every $x \in X$ there are $d_{1}, d_{2} \in D$ such that $d_{1} \preceq x \preceq d_{2}$;

- lower (upper) semicontinuous if for every $x \in X$ the subsets $\{y \in$ $X ; y \preceq x\} \quad(\{y \in X ; x \preceq y\})$ are closed with respect to the metric topology;

- continuous if it is both lower and upper semicontinuous;

- planar if $X / \sim$, the totally ordered quotient space under the indifference relation, is order-isomorphic to a non-representable subset of the lexicographic plane.

We conclude this section with a lemma that shall be used later:

Lemma 2.1. Every planar totally preordered set $(X, \preceq)$ is countably bounded. 
Proof. Without loss of generality, let us prove this assertion for a subset $\left(A, \leq_{l}\right)$ of the lexicographic plane: Let $\pi(A)=\{r \in \mathbb{R}$ : there exists $s \in \mathbb{R}$ such that $(r, s) \in A\}$. Let $D_{A} \subseteq \pi(A)$ be a countable subset of $\pi(A)$, coinitial and cofinal with respect to the usual Euclidean order on $\pi(A) \subseteq \mathbb{R}$. For every $b \in D_{A}$, let $S(b)=\{s \in \mathbb{R}:(b, s) \in A\}$. Let $D_{S(b)} \subseteq S(b)$ be a countable subset of $S(b)$, coinitial and cofinal with respect to the usual Euclidean order on $S(b) \subseteq \mathbb{R}$. Observe, finally that the countable set $\left\{(b, c): b \in D_{A}, c \in D_{S(b)}\right\}$ bounds the lexicographic order $\preceq_{l}$ on $A$.

\section{A construction of semicontinuous planar total preorders on non-separable metric spaces}

Let us now present the main result of the paper.

Theorem 3.1. Every non-separable full metric space $(X, d)$ can be endowed with a semicontinuous total preorder that is order-isomorphic to a non-representable subset of the lexicographic plane.

Proof. We provide the proof for the case of lower semicontinuity. Upper semicontinuity is handled in a similar fashion.

The strategy of the proof consists in defining both, a non-representable subset of the lexicographic plane, say $D$, and a suitable onto function $u: X \longrightarrow D$ in such a way that for every $(a, b) \in D$ the inverse image of the set $\{z \in$ $\left.D ;(a, b)<_{l} z\right\}$ under $u$, is an open subset of $X$. Then considering the binary relation on $X$ given by $x \preceq y \Longleftrightarrow u(x) \leq_{l} u(y)$, we obtain the desired conclusion.

Note that this binary relation, which obviously is a total preorder, does not admit a real-valued order-preserving representation. (If there were a realvalued order-preserving function representing $\preceq$ then, since $(X / \sim, \preceq)$ is orderisomorphic to $\left(D, \leq_{l}\right)$ (via $\left.u\right),\left(D, \leq_{l}\right)$ would be representable which is a contradiction).

In order to exhibit such a function $u$, we will make use of the following technical fact:

Every non-separable metric space $(X, d)$ admits an uncountable family of pairwise disjoint closed balls with a fixed strictly positive radius.

(See, e.g., Problem 4.A in Engelking [21]).

Suppose that, for our metric space, this radius is $\epsilon>0$. Let us denote by $E$ an uncountable subset of $X$ which consists of a suitable collection of centers of those closed balls in such a way that there is a bijection $\phi: E \longrightarrow F, F$ being an uncountable subset of $[0, \infty)$.

Let us consider the following subset of the lexicographic plane

$$
D=\{(0,0)\} \cup\{(x, y) ; x \in F, x>0,0 \leq y \leq \epsilon\} \cup\left\{(x, 0) ; x \notin F, 0<x<x^{\prime}, x^{\prime} \in F\right\} .
$$


Note that, equipped with the lexicographic total order, $D$ has a least element; namely, $(0,0)$.

Moreover, $\left(D, \leq_{l}\right)$ is not representable:

Since for a totally preordered set the existence of a representation through a real-valued order-preserving function amounts to order separability (see, e.g., Th. 1.4.8 in Bridges and Mehta [12]), an easy way to see this consists in checking that $\left(D, \leq_{l}\right)$ fails to be order separable. But this follows from the fact that $\left(D, \leq_{l}\right)$ contains an uncountable number of (non-degenerated) pairwise disjoint order intervals: To see this, for every $x \in F, x>0$ just consider the order interval $((x, 0),(x, \epsilon)))$.

For every $(x, 0) \in D, x \in F, x>0$, denote by $J(x)$ the set which consists of the union of the two segments, the horizontal one joining $(0,0)$ to $(x, 0)$ and the vertical one that connects $(x, 0)$ and $(x, \epsilon)$. Clearly, for every $x \in F, x>0$, there is a continuous and order-preserving (and therefore, homeomorphism) function from the real interval $[0, \epsilon]$ onto $J(x)$. Denote by $r_{x}(\cdot), x \in F, x>0$, such a function. Note that $r_{x}(0)=(0,0)$ for every $x \in F, x>0$.

We are now ready to define the function $u: X \longrightarrow D$ as follows:

$$
u(x)= \begin{cases}r_{\phi(e)}(\epsilon-d(x, e)), & x \in B(e, \epsilon), e \in E ; \\ (0,0), & x \in X \backslash \bigcup_{e \in E} B(e, \epsilon) .\end{cases}
$$

Since $(X, d)$ is a full metric space it holds that, for each fixed $e \in E,\{\lambda \in$ $\mathbb{R} ; \lambda=\epsilon-d(x, e), x \in B(e, \epsilon)\}=[0, \epsilon]$. This remark, together with the fact that $r_{\phi(e)}(\cdot)$ fills the set $J(\phi(e))$, shows that $\left\{d \in \mathbb{R}^{2} ; d=r_{\phi(e)}(\epsilon-d(x, e)), e \in\right.$ $E, x \in B(e, \epsilon)\}=D$. So, the image of $u$ exhausts $D$ and therefore $u$ is onto. Let $(a, b) \in D$ and denote by $G_{(a, b)}=\left\{z \in D ;(a, b)<_{l} z\right\}$. It remains to prove that the inverse image of $G_{(a, b)}$ under $u, u^{-1}\left(G_{(a, b)}\right)$, is an open subset of $X$. Two cases need to be distinguished:

(i) If $a=0$, then $(a, b)=(0,0)$ and $u^{-1}\left(G_{(0,0)}\right)=\bigcup_{e \in E} B(e, \epsilon)^{\circ}$, where " $A^{\circ}$ " denotes the interior set of A. So, $u^{-1}\left(G_{(0,0)}\right)$ is open because it is the union of open balls.

(ii) If $a>0$, then two situations can happen. On the one hand, if $a \in F$, then there is $e^{\prime} \in E$ such that $\phi\left(e^{\prime}\right)=a$. Let now $k^{\prime} \in[0, \epsilon]$ such that $r_{\phi\left(e^{\prime}\right)}\left(k^{\prime}\right)=(a, b)$. Such a $k^{\prime}$ exists because $r_{\phi\left(e^{\prime}\right)}(\cdot)$ exhausts $J\left(\phi\left(e^{\prime}\right)\right)$. So, $u^{-1}\left(G_{(a, b)}\right) \bigcap B\left(e^{\prime}, \epsilon\right)=\left\{x \in B\left(e^{\prime}, \epsilon\right) ; d\left(x, e^{\prime}\right)<\epsilon-k^{\prime}\right\}=B\left(e^{\prime}, \epsilon-k^{\prime}\right)^{\circ}$.

If $e \in E$ is such that $\phi(e)>a$, then arguing as above, there is $k \in[0, \epsilon]$, which depends on $e$, such that $r_{\phi(e)}(k)=(a, 0)$. Then $u^{-1}\left(G_{(a, b)}\right) \bigcap B(e, \epsilon)$ $=B(e, \epsilon-k)^{\circ}$. Note that,

$$
\begin{aligned}
u^{-1}\left(G_{(a, b)}\right) & =\left(u^{-1}\left(G_{(a, b)}\right) \bigcap B\left(e^{\prime}, \epsilon\right)\right) \bigcup_{\phi(e)>a}\left(u^{-1}\left(G_{(a, b)}\right) \bigcap B(e, \epsilon)\right) \\
& =B\left(e^{\prime}, \epsilon-k^{\prime}\right)^{\circ} \bigcup_{\phi(e)>a} B(e, \epsilon-k)^{\circ}
\end{aligned}
$$

which is a union of open balls, hence an open subset of $X$. 
On the other hand, if $a \notin F$, then $b=0$ and note that $\{e \in E ; \phi(e)>a\} \neq \emptyset$. In a similar way as above, for every $e \in E$ such that $\phi(e)>a$, there is $k \in[0, \epsilon]$ such that $r_{\phi(e)}(k)=(a, 0)$. Then $u^{-1}\left(G_{(a, 0)}\right) \cap B(e, \epsilon)=B(e, \epsilon-k)^{\circ}$. So, $u^{-1}\left(G_{(a, 0)}\right)=\bigcup_{\phi(e)>a}\left(u^{-1}\left(G_{(a, b)}\right) \cap B(e, \epsilon)\right)=\bigcup_{\phi(e)>a} B(e, \epsilon-k)^{\circ}$, which is again a union of open balls, hence an open subset of $X$, and the proof is complete.

Corollary 3.2. Every non-separable connected metric space can be endowed with a semicontinuous planar total preorder.

Proof. The statement follows from the evident fact that every connected metric space is full. Indeed, let $x, y \in X$ and $\delta \in \mathbb{R}$ with $0<\delta<d(x, y)$. Since the function $y \in X \longrightarrow f(y)=d(x, y) \in[0, \infty)$ is a continuous one and $X$ is connected, it holds that $f(X)$ is a connected subset of the reals; i.e., it is a real interval. So, there is $z \in X$ such that $d(x, z)=\delta$ and therefore $(X, d)$ is a full metric space.

Remark 3.3. A full metric space may fail to be connected. An example is $X=$ $(-\infty, 0) \cup[3,+\infty) \subset \mathbb{R}$ endowed with the usual Euclidean topology of the real line, that is obviously metric. Notice also that this space is totally ordered in the natural way, and endowed with the order topology it is indeed connected and topologically isomorphic to the whole real line. Hence, $X$ is metric as regards the order topology. As a matter of fact, the Euclidean topology on $X$ does not coincide with that order topology. The coincidence of the inherited Euclidean topology and the order topology on subsets of the real line, or more generally of Euclidean spaces, is a classical problem in the theory of representation of totally ordered structures (also known as "Utility Theory" in contexts of Economics). For a further reading in this direction, see Candeal and Induráin [17], Beardon $[3,4]$ and Candeal et al. [19].

Remark 3.4. It is important to emphasize some facts in order to clarify the content of the previous Theorem 3.1. The abstract metric space involved in the statement of the theorem is not necessarily a coordinate space (like, e.g., $\left.l_{\infty}\right)$. If, for instance, we were assuming that the metric space is $l_{\infty}$, then the semicontinuous planar total preorder provided by the theorem has nothing to do with the natural lexicographic total order defined on $l_{\infty}$. Note that the usual (coordinatewise) lexicographic total order on $l_{\infty}$ is not, either upper or lower, semicontinuous with respect to the metric (norm) topology on $l_{\infty}$.

Remark 3.5. In Corollary 4.5 in Bosi and Herden [9] it was already observed that a metric space is non-separable if and only if there exists an upper semicontinuous non-representable total preorder definable on such space. However the proof of that result uses the theorem by Estévez and Hervés [22] mentioned in the Introduction, which is based on long chains instead of planar chains. 
In general, semicontinuity cannot be replaced by continuity in the statement of the previous Theorem 3.1. Actually, we have the following impossibility result:

Theorem 3.6. No continuous planar total preorder can be defined on a pathconnected metric space $(X, d)$.

Proof. This assertion follows from a theorem by Monteiro [29], that states that any continuous and countably bounded total preorder defined on a pathconnected metric space $(X, d)$ is always representable. But, by Lemma 2.1, any planar total preorder on $(X, d)$ is countably bounded. Therefore, it would be representable by Monteiro's theorem. This contradiction proves that no continuous lexicographic preorder on $(X, d)$ can exist.

Remark 3.7. Indeed, this result could still be generalized to the class of separably connected metric spaces, wider than that of (just) path connected (see Corollary 3 in Candeal et al. [16], and Theorem 3.3 in Campión et al. [14]). A topological space $(X, \tau)$ is said to be separably connected, if for every two points $x, y \in X$ there exists a connected and separable subset $C \subseteq X$ that includes $x$ and $y$.

Final Remark. We leave as open problems the following ones:

(1) Study, on nonseparable metric spaces, the singularity of second type (Aronszajn) with respect to continuous and semicontinuous ordinal representability.

(2) Study, on nonseparable general metric spaces (not only connected ones!) the singularity of third type (planar) with respect to continuous and semicontinuous ordinal representability.

(3) Study on general topological spaces the different three kinds of singularity with respect to continuous and semicontinuous ordinal representability.

Acknowledgements. Thanks are given to an anonymous referee for her/his valuable suggestions and comments. This work has been supported by the research projects MTM 2006 - 15025 and MTM2007 - 62499, of the Spanish Ministry of Culture. We want to express our gratitude to the co-participants in the research projects J. R. De Miguel, O. R. García-Catalán, M. C. Leránoz, and M. Zudaire (UPNA. Pamplona, Spain) for their suggestions and comments on previous drafts of the manuscript.

\section{References}

[1] C. D. Aliprantis and K. C. Border, Infinite-Dimensional Analysis: A Hitchhiker's Guide, Second edition, Springer-Verlag, Berlin, 1999.

[2] C. D. Aliprantis, D. J. Brown, and O. Burkinshaw, Existence and Optimality of Competitive Equilibria, Springer-Verlag, Berlin, 1990.

[3] A. F. Beardon, Totally ordered subsets of Euclidean space, J. Math. Econom. 23 (1994), no. $4,391-393$. 
[4] Utility theory and continuous monotonic functions, Econom. Theory 4 (1994), no. $4,531-538$

[5] A. F. Beardon, J. C. Candeal, G. Herden, E. Induráin, and G. B. Mehta, The nonexistence of a utility function and the structure of non-representable preference relations, J. Math. Econom. 37 (2002), no. 1, 17-38.

[6] Lexicographic decomposition of chains and the concept of a planar chain, J. Math. Econom. 37 (2002), no. 2, 95-104.

[7] T. Bewley, Existence of equilibria in economies with infinitely many commodities, J. Econom. Theory 4 (1972), no. 3, 514-540.

[8] G. Bosi, J. C. Candeal, and E. Induráin, Continuous representability of homothetic preferences by means of homogeneous utility functions, J. Math. Econom. 33 (2000), no. 3, 291-298.

[9] G. Bosi and G. Herden, On the structure of completely useful topologies, Appl. Gen. Topol. 3 (2002), no. 2, 145-167.

[10] On a strong continuous analogue of the Szpilrajn theorem and its strengthening by Dushnik and Miller, Order 22 (2005), no. 4, 329-342.

[11] On a possible continuous analogue of the Szpilrajn theorem and its strengthening by Dushnik and Miller, Order 23 (2006), no. 4, 271-296.

[12] D. S. Bridges and G. B. Mehta, Representations of Preferences Orderings, Lecture Notes in Economics and Mathematical Systems, 422. Springer-Verlag, Berlin, 1995.

[13] M. J. Campión, J. C. Candeal, A. S. Granero, and E. Induráin, Ordinal representability in Banach spaces, Methods in Banach space theory, 183-196, London Math. Soc. Lecture Note Ser., 337, Cambridge Univ. Press, Cambridge, 2006.

[14] M. J. Campión, J. C. Candeal, and E. Induráin, On Yi's extension property for totally preordered topological spaces, J. Korean Math. Soc. 43 (2006), no. 1, 159-181.

[15] _ The existence of utility functions for weakly continuous preferences on a Banach space, Math. Social Sci. 51 (2006), no. 2, 227-237.

[16] J. C. Candeal, C. Hervés, and E. Induráin, Some results on representation and extension of preferences, J. Math. Econom. 29 (1998), no. 1, 75-81.

[17] J. C. Candeal and E. Induráin, Utility functions on chains, J. Math. Econom. 22 (1993), no. $2,161-168$.

[18] _ Lexicographic behaviour of chains, Arch. Math. (Basel) 72 (1999), no. 2, 145152

[19] J. C. Candeal, E. Induráin, and G. B. Mehta, Further remarks on totally ordered representable subsets of Euclidean space, J. Math. Econom. 25 (1996), no. 4, 381-390.

[20] _ Order preserving functions on ordered topological vector spaces, Bull. Austral. Math. Soc. 60 (1999), no. 1, 55-65.

[21] R. Engelking, Outline of General Topology, Translated from the Polish by K. Sieklucki North-Holland Publishing Co., Amsterdam; PWN-Polish Scientific Publishers, Warsaw; Interscience Publishers Division John Wiley \& Sons, Inc., New York 1968.

[22] M. Estévez and C. Hervés, On the existence of continuous preference orderings without utility representations, J. Math. Econom. 24 (1995), no. 4, 305-309.

[23] A. Giarlotta, Representable lexicographic products, Order 21 (2004), no. 1, 29-41.

[24] - The representability number of a chain, Topology Appl. 150 (2005), no. 1-3, $157-177$.

[25] G. Herden and A. Pallack, Useful topologies and separable systems, Appl. Gen. Topol. 1 (2000), no. 1, 61-82.

[26] R. Isler, Semicontinuous utility functions in topological spaces, Riv. Mat. Sci. Econom. Social. 20 (1997), no. 1, 111-116.

[27] A. Mas-Colell, Papers presented at the Colloquium on Mathematical Economics, J. Math. Econom. 2 (1975), no. 2, 263-295. 
[28] A. Mas-Colell and W. R. Zame, Equilibrium theory in infinite-dimensional spaces, Handbook of mathematical economics, Vol. IV, 1835-1898, Handbooks in Econom., 1, NorthHolland, Amsterdam, 1991.

[29] P. K. Monteiro, Some results on the existence of utility functions on path connected space, J. Math. Econom. 16 (1987), no. 2, 147-156.

[30] A. J. Ostaszewski, On the descriptive set theory of the lexicographic square, Fund. Math. 87 (1975), no. 3, 261-281.

[31] T. Rader, The existence of a utility function to represent preferences, Review of Economic Studies 30 (1963), 229-232.

[32] S. Romaguera and M. Sanchis, Applications of utility functions defined on quasi-metric spaces, J. Math. Anal. Appl. 283 (2003), no. 1, 219-235.

[33] L. A. Steen and J. A. Seebach Jr., Counterexamples in Topology, Dover, New York, 1978.

[34] G. Yi, Continuous extension of preferences, J. Math. Econom. 22 (1993), no. 6, 547-555.

MARÍA JESÚS CAMPIÓN

Universidad Pública de Navarra

Departamento de Matemáticas

Campus Arrosadía

E-31006. PAMPlona, Spain

E-mail address: mjesus.campion@unavarra.es

Juan Carlos Candeal

UNIVERSIDAD DE ZARAGOZA

Facultad de Ciencias Económicas y Empresariales

Departamento de Análisis Económico

C/ Doctor Cerrada 1-3. E-50005. Zaragoza, Spain

E-mail address: candeal@unizar.es

ESTEBAN INDURÁIN

Universidad PÚblica de Navarra

Departamento de Matemáticas

Campus Arrosadía. E-31006. Pamplona, Spain

E-mail address: steiner@unavarra.es 